\title{
Characterizations of classes of risk measures by dispersive orders
}

\author{
Miguel A. Sordo* \\ Departamento de Estadística e Investigación Operativa \\ Facultad de Ciencias Económicas y Empresariales \\ Universidad de Cádiz \\ 11002 Cádiz, Spain. \\ Tel.: +34-956-015403; fax: +34-956-016288 \\ E-mail address: mangel.sordo@uca.es
}

\begin{abstract}
In this paper, a class $C_{1}$ of risk measures, which generalizes the class of risk measures for the right-tail deviation suggested by Wang (1998), is characterized in terms of dispersive order. If dispersive order does not hold, unanimous comparisons are still possible by restricting our attention to a subclass $C_{2} \subset C_{1}$ and then the criterion is the excess wealth order. Sufficient conditions for stochastic equivalence of excess wealth ordered random variables are derived in terms of some particular measures of $C_{2}$.
\end{abstract}

MSC: IM30

Keywords: dispersive order, excess wealth order, risk measures, Gini mean difference, Wang's right-tail deviation.

* Supported by Ministerio de Educación y Ciencia (grant SEJ2005-06678). Please, cite this paper as follows: Sordo, M. A. (2008). Characterizations of classes of risk measures by dispersive orders. Insurance: Mathematics and Economics 42, 1028-1034. 


\section{Introduction}

In actuarial science, special efforts have been made to measure the risk associated with large-loss events. An important reason for measuring the right-tail risk is to make risk comparisons and, for this purpose, stochastic orders, which require unanimous agreement among classes of risk measures, can be used. For actuarial applications of stochastic orders, we refer to the books of Goovaerts et al. (1990) and Kaas et al. (1994). For a general overview of this topic, see the monograph of Shaked and Shanthikumar (2006).

In this paper, we characterize two nested classes of risk measures, $C_{1}$ and $C_{2}$, which include a number of well-known measures, in terms of dispersive order and excess wealth order, respectively. In order to introduce these classes, consider a random variable $X$ with distribution function $F$ and let $F^{-1}$ be the corresponding quantile function, defined by $F^{-1}(t)=\inf \{x: F(x) \geq t\}$. Let $a:[0,1] \longmapsto[0,1]$ be a distortion function, that is, an increasing function that satisfies $a(0)=0$ and $a(1)=1$. In the context of the premium principle of Wang (1996), the expectation with respect to the distorted probability $a \circ P$, defined by

$$
E_{a}(X)=\int_{-\infty}^{0}\{a(P[X>t])-1\} d t+\int_{0}^{\infty} a(P[X>t]) d t
$$

represents the certainty equivalent to risk $X$, that is, the market price for transferring the risk $X$. Wang (1998) derives a general class of risk measures for the right-tail deviation from the difference between the certainty equivalent and the expected loss:

$$
\rho_{a}(X)=E_{a}(X)-E(X),
$$

where $a$ is a concave distortion function. As shown by Jones and Zitikis (2003), if $a$ is piecewise differentiable and $A(t)=1-a(1-t)$, then $E_{a}(X)=$ $E_{A}(X)$, where

$$
E_{A}(X)=\int_{0}^{1} F^{-1}(t) d A(t) .
$$

Taking into account that $a$ is a concave distortion function if, and only if, $A$ is a convex distortion function, it follows that each $\rho_{a}(X)$ of the form $(1)$ can be expressed using the alternative representation

$$
\rho_{A}(X)=\int_{0}^{1} F^{-1}(t) d A(t)-E(X)
$$


where $A(t)$ is a convex distortion function. A number of well-known risk measures can be expressed under the form (3), including the right-tail deviation suggested by Wang (1998), given by (3) with $A(t)=1-\sqrt{1-t}$ (see Jones and Zitikis, 2003) and the Gini's mean semidifference, given by (3) with $A(t)=t^{2}$ (see Nygard and Sandström, 1981).

Note that (3) involves dispersion about the mean of the distribution. However, a number of authors, including Bickel and Lehmann (1979), Quiggin (1982), Muñoz-Pérez (1990), Landsberger and Meilijson (1994a,b), Shaked and Shanthikumar (1998), Fernández-Ponce et al. (1998), and Chateauneuf et al. (2004, 2005), instead of looking at dispersion relative to a fixed point, consider the spread of a random variable throughout its distribution. We follow this approach by focusing on a class $C_{1}$ of risk measures of the type

$$
\rho_{A, B}(X)=\int_{0}^{1} F^{-1}(t) d A(t)-\int_{0}^{1} F^{-1}(t) d B(t)
$$

where $A(t)$ and $B(t)$ are two distortion functions such that $A B^{-1}(t)$ is convex. The convexity of $A B^{-1}(t)$ ensures that $\rho_{A, B}(X) \geq 0$ and allows us to view (4) as a generalization of (3), which is obtained with $B(t)=t$. Jones and Zitikis (2003) note that a number of risk measures, originally expressed using distorted probabilities, take the form (4) for adequate choices of $A(t)$ and $B(t)$. This representation of risk measures is very useful, because empirical estimators of quantities with the form (4) can be obtained using the theory of $L$-statistics, which are linear combinations of order statistics; see Jones and Zitikis $(2003,2007)$ and Jones et al. (2006), for details. Members of $C_{1}$ satisfy the next intuitively desirable properties for a measure of variability (see Bickel and Lehmann, 1979; we refer to Rockafellar et al., 2006, for an interpretation and discussion of these properties in an actuarial context).

Theorem 1 Each functional $\rho_{A, B} \in C_{1}$ has the following properties:

(i) $\rho_{A, B}(X+c)=\rho_{A, B}(X)$ for all $c$,

(ii) $\rho_{A, B}(c X)=c \rho_{A, B}(X)$ for all $c>0$,

(iii) $\rho_{A, B}(c)=0$ for any degenerate random variable at $c$,

(iv) $\rho_{A, B}(X) \geq 0$ for all random variable $X$,

(v) $\rho_{A, B}(X+Y)=\rho_{A, B}(X)+\rho_{A, B}(Y)$ for $X$ and $Y$ comonotonic.

Proof. Properties $(i)-($ iii $)$ are easy to see. In order to prove $(i v)$, note that if $A$ and $B$ are distortion functions and $A B^{-1}$ is convex, then $A(t) \leq B(t)$ 
and, therefore,

$$
\rho_{A, B}(X)=\int_{0}^{1} F^{-1}(t) d[A(t)-B(t)]=\int_{0}^{1}[B(t)-A(t)] d F^{-1}(t) \geq 0 .
$$

Finally, $(v)$ follows from the fact that comonotonicity implies additivity of the certainty equivalents (Wang, 1996).

The class $C_{1}$ includes a number of measures of interest not having the form (3). One of them is the Wang's (1998) two-sided deviation, given by (4) with $A(t)=1-\sqrt{1-t}$ and $B(t)=\sqrt{t}$ (see Jones and Zitikis, 2003). In order to provide another example, let $X_{1}, \ldots X_{n}$ be $n$ independent copies of a non-negative random variable $X$ and denote their corresponding order statistics by $X_{1: n}, \ldots, X_{n: n}$. An intuitive measure for the right-tail of $X$ is the mean of the last sample spacing ${ }^{1}$, given by

$$
E\left[X_{n: n}-X_{n-1: n}\right]=n \int_{0}^{\infty} F^{n-1}(x) \bar{F}(x) d x
$$

where $\bar{F}=1-F$ (Kendall and Stuart, 1977, p.368). By making the change of variable $F(x)=t$, we see, via integration by parts, that (5) belongs to $C$ (up to a scale factor) with $A(t)=t^{n}$ and $B(t)=t^{n-1}$. A third example is, for each $0<p<1$, the measure

$$
E S F_{p}(X)=E\left[\left(X-F^{-1}(p)\right)^{+}\right]=\int_{p}^{1}\left(F^{-1}(t)-F^{-1}(p)\right) d t,
$$

which is called, in actuarial literature ${ }^{2}$, the expected shortfall at level $p$ (see Dhaene et al., 2006, 2007) and represents the expected shortfall of the portfolio with loss $X$ and solvency capital requirement $F^{-1}(p)$. Note that (6) belongs to $C_{1}$ with

$$
A(t)=\max \left(0, \frac{t-p}{1-p}\right) \text { and } B(t)= \begin{cases}0 & \text { if } t<p \\ 1 & \text { if } t \geq p\end{cases}
$$

(the relevant function $A B^{-1}(t)$ is constantly equal to 0 ).

\footnotetext{
${ }^{1}$ This measure is also of great interest in auction theory, where, for a buyer's auction, it represents the expected rent with $n$ bidders (see Li, 2005, and Kochar et al., 2007).

${ }^{2}$ The function $E S F_{p}(X)$ is known under different names in other fields, such as the excess wealth transform in the theory of wealth inequality and the right spread function in reliability theory.
} 
The class $C_{1}$ not only unifies a number of risk measures but also provides a link between the measures and stochastic orders. In Section 2, we characterize the comparison of random variables according to the measures $\rho_{A, B}$ of $C_{1}$ in terms of the well-known dispersive order (Doksum, 1969; Bickel and Lehmann, 1979), whose definition is recalled here.

Definition 2 Let $X$ and $Y$ be two random variables with respective distribution functions $F$ and $G$. Then, $X$ is said to be smaller than $Y$ in the dispersive order (denoted by $X \leq_{\text {disp }} Y$ ) if

$$
F^{-1}(p)-F^{-1}(q) \leq G^{-1}(p)-G^{-1}(q), \text { for all } 0<q<p<1 .
$$

From this characterization, we deduce that if $X$ and $Y$ are ordered in the dispersive order, then we can judge between them according to any measures $\rho_{A, B}$ without needing to agree on the form of $A(t)$ and $B(t)$ (except that $A B^{-1}(t)$ is convex). However, although dispersive order provides an important tool for risk comparisons, (8) is a strong requirement and many pair of distributions can fail to satisfy it. This justifies the convenience, from the perspective of both decision-makers and empirical researchers, of employing a weaker order to compare risks. We also prove in Section 2 that, when dispersive order does not hold, unanimous comparisons are still possible by restricting our attention to the class

$$
C_{2}=\left\{\text { measures of } C_{1} \text { with } B \text { convex }\right\}
$$

It is straightforward to show that each member of $C_{2}$ is a difference between two parameters to the right of the mean. Therefore, a decision-marker who employs $\rho_{A, B}$ in $C_{2}$ (for example, (5)) is more sensitive to the variability within the right-tail of the distribution that one who employs $\rho_{A, B}$ in $C_{1}$ (but not in $C_{2}$ ). The criterion to obtain unanimous comparisons according to $C_{2}$ is the so-called excess wealth order, whose definition is recalled here (Fernández-Ponce et al., 1998; Shaked and Shanthikumar, 1998).

Definition 3 Let $X$ and $Y$ be two random variables with distribution functions $F$ and $G$, respectively. Then, we say that $X$ is smaller than $Y$ in the excess wealth order (denoted by $X \leq_{e w} Y$ ) if $E S F_{p}(X) \leq E S F_{p}(Y)$, for all $p \in$ $(0,1)$. 
The plan of the paper is as follows. The main characterizations are stated in Section 2. In Section 3 we prove that if two random variables $X$ and $Y$ are ordered in the excess wealth order and $\rho_{A, B}(X)=\rho_{A, B}(Y)$ for some special $\rho_{A, B} \in C_{2}$, then they are identically distributed or they differ by a location parameter. Section 4 provides conclusions.

In this paper, "increasing" and "decreasing" mean "non-decreasing" and "non-increasing", respectively. We shall be assuming throughout this paper that all the random variables under consideration have finite means.

\section{The characterizations}

Throughout this section, let $\Omega_{1}$ denote the class of distortion functions and let $\Omega_{2}$ denote the class of convex distortion functions. In order to obtain the main characterizations, we first prove that each $\rho_{A, B} \in C_{i}(i=1,2)$ with $B$ non-degenerate ${ }^{3}$, can be expressed as a weighted area under the curve

$$
V_{B}(X, p)=\int_{p}^{1}\left[F^{-1}(t)-E_{B}(X)\right] d B(t), p \in(0,1),
$$

where

$$
E_{B}(X)=\int_{0}^{1} F^{-1}(t) d B(t) .
$$

Functionals of the form (10) include parameters like the mean (obtained when $B(t)=t$ ) and, more generally, the trimmed expectations, defined by

$$
\frac{1}{\beta-\alpha} \int_{\alpha}^{\beta} F^{-1}(t) d t, \quad 0<\alpha<\beta<1,
$$

obtained when $B$ is the distribution function of a uniform random variable on the interval $(\alpha, \beta)$. Therefore, for each $B$ fixed, (9) can be considered as a measure of variability to the right of $F^{-1}(p)$. Observe that, for each distribution function $B, V_{B}(X, p)$ is a continuous function in $p$ on $(0,1)$ with

$$
V_{B}(X, 0)=V_{B}(X, 1)=0 .
$$

In addition, it is easy to see that $V_{B}(X, p)$ is increasing for $0 \leq p \leq$ $F\left(E_{B}(X)\right)$ and decreasing for $F\left(E_{B}(X)\right) \leq p \leq 1$, therefore

$$
V_{B}(X, p) \geq 0 \text { for all } p \in(0,1) \text {. }
$$

\footnotetext{
${ }^{3}$ If $B$ is degenerate, as in $(7)$, then $V_{B}(X, p)=0$ for all $p$.
} 
Lemma 4 Let $\rho_{A, B}$ be a functional of the form (4) with $B \in \Omega_{i}(i=1,2)$, such that $B$ is non-degenerate. Then, $\rho_{A, B} \in C_{i}(i=1,2)$, if, and only if, there exist an increasing function $\alpha$ and such that

$$
\rho_{A, B}(X)=\int_{0}^{1} V_{B}(X, t) d \alpha(t) .
$$

Proof. Assume that $\rho_{A, B} \in C_{i}(i=1,2)$. Using the notation (10) we have

$$
\rho_{A, B}(X)=\int_{0}^{1}\left[F^{-1}(t)-E_{B}(X)\right] d A(t)
$$

where $A B^{-1}$ is convex and $B \in \Omega_{i}(i=1,2)$. Note that $A B^{-1}(t)$ is convex if, and only if, $\alpha(t)=\frac{d A(t)}{d B(t)}$ is increasing. Therefore, (15) can be rewritten as

$$
\begin{aligned}
\rho_{A, B}(X) & =\int_{0}^{1} \alpha(t)\left[F^{-1}(t)-E_{B}(X)\right] d B(t) \\
& =-\int_{0}^{1} \alpha(t) d V_{B}(X, t),
\end{aligned}
$$

with $\alpha$ increasing. Integration by parts of (16) and (12) yield

$$
\rho_{A, B}(X)=\int_{0}^{1} V_{B}(X, t) d \alpha(t),
$$

as required. The converse is proved similarly.

In order to obtain the next result, we will exploit the well-known relationships between dispersive order and stochastic order (denoted by $\leq_{s t}$ ) and between excess wealth order and increasing convex order (denoted by $\leq_{i c x}$ ). Sordo and Ramos (2007) show that the orders $\leq_{s t}$ and $\leq_{i c x}$ can be characterized as follows.

Theorem 5 Let $X$ and $Y$ be two random variables with distribution functions $F$ and $G$, respectively. Then,

(i) $X \leq_{s t} Y$ if, and only if, $E_{B}(X) \leq E_{B}(Y)$ for all $B \in \Omega_{1}$.

(ii) $X \leq_{i c x} Y$ if, and only if, $E_{B}(X) \leq E_{B}(Y)$ for all $B \in \Omega_{2}$.

The following results are due to Muñoz-Pérez (1990) and Belzunce (1999). 
Theorem $6^{4}$ Let $X$ and $Y$ be two random variables with distribution functions $F$ and $G$, respectively. Then,

(i) $X \leq_{\text {disp }} Y \leftrightarrow\left(X-F^{-1}(p)\right)^{+} \leq_{s t}\left(Y-G^{-1}(p)\right)^{+}$for all $p \in(0,1)$.

(ii) $X \leq_{e w} Y \leftrightarrow\left(X-F^{-1}(p)\right)^{+} \leq_{i c x}\left(Y-G^{-1}(p)^{+}\right.$, for all $p \in(0,1)$.

Now, we are in conditions to prove the following theorem.

Theorem 7 Let $X$ and $Y$ be two random variables with distribution functions $F$ and $G$, respectively. Then,

(i) If $X \leq_{\text {disp }} Y$, then $V_{B}(X, p) \leq V_{B}(Y, p)$, for all $p \in(0,1)$, for all $B \in \Omega_{1}$.

(ii) $X \leq_{e w} Y$ if, and only if, $V_{B}(X, p) \leq V_{B}(Y, p)$, for all $p \in(0,1)$, for all $B \in \Omega_{2}$.

Proof. (i) Suppose that $X \leq_{\text {disp }} Y$. Equivalently, by Theorem $6(i)$ we have that

$$
\left(X-F^{-1}(p)\right)^{+} \leq_{s t}\left(Y-G^{-1}(p)^{+} \text {, for all } p \in(0,1) .\right.
$$

For each $p \in(0,1)$, let $F_{p}^{-1}(t)$ be the quantile function associated to the random variable $\left(X-F^{-1}(p)\right)^{+}$. Taking into account that

$$
F_{p}^{-1}(t)=\left\{\begin{array}{cl}
0 & \text { if } t<p \\
F^{-1}(t)-F^{-1}(p) & \text { if } t \geq p
\end{array},\right.
$$

it follows from Theorem 5(i) that (18) is equivalent to

$$
\begin{gathered}
\int_{p}^{1}\left[F^{-1}(t)-F^{-1}(p)\right] d B(t) \leq \int_{p}^{1}\left[G^{-1}(t)-G^{-1}(p)\right] d B(t), \\
\forall p \in(0,1), \forall B \in \Omega_{1} .
\end{gathered}
$$

Condition (19) can be rewritten as

$$
\begin{gathered}
\int_{p}^{1}\left[F^{-1}(t)-G^{-1}(t)\right] d B(t) \leq(1-B(p))\left(F^{-1}(p)-G^{-1}(p)\right), \\
\forall p \in(0,1), \forall B \in \Omega_{1} .
\end{gathered}
$$

\footnotetext{
${ }^{4}$ Belzunce (1999) obtained (ii) for continuous random variables, but it can be shown that the continuity assumption is no necessary.
} 
By differentiation, it can be verified that the latter is equivalent to say that

$$
\frac{\int_{p}^{1}\left[F^{-1}(t)-G^{-1}(t)\right] d B(t)}{\int_{p}^{1} d B(t)} \text { is decreasing in } p \in(0,1), \forall B \in \Omega_{1} .
$$

From (20) it follows that

$$
\begin{gathered}
\frac{\int_{p}^{1}\left[F^{-1}(t)-G^{-1}(t)\right] d B(t)}{\int_{p}^{1} d B(t)} \leq \int_{0}^{1}\left[F^{-1}(t)-G^{-1}(t)\right] d B(t), \\
\forall p \in(0,1), \forall B \in \Omega_{1} .
\end{gathered}
$$

Since

$$
E_{B}(X)-E_{B}(Y)=\int_{0}^{1}\left[F^{-1}(t)-G^{-1}(t)\right] d B(t)
$$

it is easy to see that (21) is equivalent to

$$
V_{B}(X, p) \leq V_{B}(Y, p), \text { for all } p \in(0,1), \forall B \in \Omega_{1} .
$$

(ii) The proof of sufficiency is analogous to the proof of part (i) but it uses Theorem 6(ii) and Theorem 5(ii) instead of Theorem 6(i) and Theorem 5(i), respectively (we omit the details). In order to prove the converse, suppose that $V_{B}(X, p) \leq V_{B}(Y, p)$, for all $p \in(0,1)$, for all $B \in \Omega_{1}$. For each $u \in(0, p)$, the distortion function defined by

$$
B_{u}(t)=\max \left(0, \frac{t-u}{1-u}\right)
$$

is convex. Hence,

$$
V_{B_{u}}(X, p) \leq V_{B_{u}}(Y, p), \text { for all } p \in(0,1),
$$

or, equivalently,

$$
\begin{gathered}
\int_{p}^{1}\left[F^{-1}(t)-E_{B u}(X)\right] d B_{u}(t) \leq \int_{p}^{1}\left[G^{-1}(t)-E_{B u}(Y)\right] d B_{u}(t) \\
\text { for all } p \in(0,1), \text { for all } u \in(0, p)
\end{gathered}
$$

Since

$$
E_{B_{u}}(X)=\frac{1}{1-u} \int_{u}^{1} F^{-1}(t) d t, u \in(0,1)
$$


and

$$
E_{B_{u}}(Y)=\frac{1}{1-u} \int_{u}^{1} G^{-1}(t) d t, u \in(0,1),
$$

(22) can be rewritten as

$$
\frac{\int_{p}^{1}\left[F^{-1}(t)-G^{-1}(t)\right] d t}{1-p} \leq \frac{\int_{u}^{1}\left[F^{-1}(t)-G^{-1}(t)\right] d t}{1-u}, \text { for all } 0<u<p<1
$$

and this is equivalent to $X \leq_{e w} Y$ (see (3.C.4) in Shaked and Shanthikumar, 2006).

In the following result, we characterize the classes $C_{1}$ and $C_{2}$ in terms of dispersive order and excess wealth order, respectively. Note that dispersive order requires the difference between any two quantiles of $X$ to be smaller than the corresponding quantiles of $Y$. As we show below, each interquantile difference is a member $\rho_{A, B}$ of $C_{1}$ with $B$ degenerate, which implies that it can not be expressed in terms of the curve $V_{B}(X, p)$ (see footnote 3 ); for this reason, the condition in Theorem $7(i)$ is necessary but not sufficient for the dispersive order.

Theorem 8 Let $X$ and $Y$ be two random variables with distribution functions $F$ and $G$, respectively. Then,

(i) $X \leq_{\text {disp }} Y$ if, and only if, $\rho_{A, B}(X) \leq \rho_{A, B}(Y)$ for all $\rho_{A, B} \in C_{1}$.

(ii) $X \leq_{e w} Y$ if, and only if, $\rho_{A, B}(X) \leq \rho_{A, B}(Y)$ for all $\rho_{A, B} \in C_{2}$.

Proof. (i) In order to prove sufficiency, assume $X \leq_{\text {disp }} Y$ and let $\rho_{A, B} \in$ $C_{1}$. First, we suppose that $B$ is non-degenerate. Then, from Lemma 4 , there exist an increasing $\alpha(t)$ such that (14) holds. Therefore, $\rho_{A, B}(X) \leq \rho_{A, B}(Y)$ or, equivalently,

$$
\int_{0}^{1} V_{B}(X, t) d \alpha(t) \leq \int_{0}^{1} V_{B}(Y, t) d \alpha(t)
$$

follows from Theorem 7(i) and the monotonic nature of $\alpha$. Now suppose that $B$ is a degenerate function given by

$$
B(t)= \begin{cases}0 & \text { if } t<p \\ 1 & \text { if } t \geq p\end{cases}
$$


This implies that $A(t)$ must be equal to zero for all $t \leq p$ since otherwise, $A B^{-1}(t)$ is not convex. Then we can write

$$
\rho_{A, B}(X)=\int_{p}^{1}\left[F^{-1}(t)-F^{-1}(p)\right] d A(t),
$$

and $\rho_{A, B}(X) \leq \rho_{A, B}(Y)$ follows from (19). Necessity follows immediately by noting that each interquantile difference

$$
F^{-1}(p)-F^{-1}(q), 0<q<p<1
$$

belongs to $C_{1}$ with

$$
A(t)=\left\{\begin{array}{ll}
0 & \text { if } t \leq p \\
1 & \text { if } t>p
\end{array}, B(t)= \begin{cases}0 & \text { if } t \leq q \\
1 & \text { if } t>q\end{cases}\right.
$$

(the relevant function $A B^{-1}(t)$ is constantly equal to 0 ).

(ii) The proof of sufficiency is analogous to the proof of part (i). In order to prove the converse, suppose that

$$
\rho_{A, B}(X) \leq \rho_{A, B}(Y) \text { for all } \rho_{A, B} \in C_{2} .
$$

The function $\alpha_{p}(t)$, defined by

$$
\alpha_{p}(t)=\left\{\begin{array}{cc}
0 & \text { if } \quad t<p \\
1 & \text { if } \quad t \geq p
\end{array}\right.
$$

is an increasing function of $t$ for each $p \in(0,1)$. Using (17), it follows from (26) that $V_{B}(X, p) \leq V_{B}(Y, p)$, for all $p \in(0,1)$, for all $B \in \Omega_{2}$, which is the same, by Theorem $7(\mathrm{ii})$, as $X \leq_{e w} Y$

\section{Conditions for stochastic equivalence un- der excess wealth order}

The purpose of this section is to provide some sufficient conditions, in terms of some special risk measures of the class $C_{2}$, for stochastic equality of two excess-wealth ordered random variables. Since dispersive order is stronger than excess wealth order, these conditions also imply stochastic equivalence of two random variables ordered in the dispersive order. Many authors have 
investigated the conditions, involving various stochastic orders, under which two ordered random variables must be stochastically equal (see, for example, Battacharjee and Sethuraman, 1990, Scarsini and Shaked, 1990, Bhattarcharjee, 1991, Jun, 1994, Li and Zhu, 1994, Cai and Wu, 1997, Denuit et al., 2000, Bhattacharjee and Bhattacharya, 2000, and Sordo and Ramos, 2007). Note that two random variables are equivalent in terms of the excess wealth order if, and only if, either they are identically distributed or they differ by a location parameter (Kochar and Carrière, 1997); therefore, the obtained conditions lead necessarily to the stochastic equivalence up to a location parameter. In the following results, " $={ }_{s t}$ " denotes equality in distribution.

Theorem 9 Let $X$ and $Y$ be two random variables with distribution functions $F$ and $G$, respectively. If $X \leq_{e w} Y$ and $\rho_{A, B}(X)=\rho_{A, B}(Y)$ for some $\rho_{A, B} \in C_{2}$ such that $d A(t) / d B(t)=\alpha(t)$ is strictly increasing on $(0,1)$, then $X \equiv_{s t} Y+c$, for some real constant $c$.

Proof. Suppose that $X \leq_{e w} Y$ or, equivalently, that

$$
V_{B}(X, p) \leq V_{B}(Y, p) \text {, for all } p \in(0,1) \text {, for all convex } B \text {. }
$$

By hypothesis, we have that

$$
\int_{0}^{1} V_{B}(X, t) d \alpha(t)=\int_{0}^{1} V_{B}(Y, p) d \alpha(t)
$$

or, equivalently, that

$$
\int_{0}^{1}\left[V_{B}(Y, t)-V_{B}(X, t)\right] d \alpha(t)=0,
$$

for some strictly increasing function $\alpha(t)$. Combining (27), (28) and the strictly monotony of $\alpha$, we assert that $V_{B}(X, t)=V_{B}(Y, t)$ for all $t \in(0,1)$. Suppose, by contradiction, that there exists an interval $\left(t_{1}, t_{2}\right) \subset(0,1)$ such that $V_{B}(X, t)<V_{B}(Y, t)$ for all $t \in\left(t_{1}, t_{2}\right)$. Then

$$
\int_{0}^{1}\left[V_{B}(Y, t)-V_{B}(X, t)\right] d \varphi(t) \geq \int_{t_{1}}^{t_{2}}\left[V_{B}(Y, t)-V_{B}(X, t)\right] d \varphi(t)>0
$$

since $\alpha$ is strictly increasing, and this contradicts (28). Therefore,

$$
V_{B}(X, t)=V_{B}(Y, t), \text { for all } t \in(0,1) \text {. }
$$


Differentiating both sides of (29) with respect to $t$ we get

$$
F_{X}^{-1}(t)=F_{Y}^{-1}(t)+c, \text { for some real } c \text { and for all } t .
$$

Finally, it is easy to see that (30) holds if, and only if, $X$ and $Y$ differ by a location parameter. Hence the result follows.

The Gini's mean semidifference, defined by

$$
G(X)=E\left[\max \left(X_{1}, X_{2}\right)\right]-\mu_{X}=\frac{E\left|X_{1}-X_{2}\right|}{2},
$$

where $X_{1}, X_{2}$ (resp. $\left.Y_{1}, Y_{2}\right)$ are independent copies of $X$ (resp. $Y$ ), plays an important role in measuring the right-tail risk (see Wang, 1998). It is well-known (Kochar and Carrière, 1997; Fernández-Ponce et al. 1998) that $X \leq_{e w} Y$ implies $G(X) \leq G(Y)$. But more than that is true. In fact, if two random variables are ordered in the excess wealth order and $G(X)=G(Y)$, then they must have the same distribution (up to a location parameter). This is stated in the next corollary.

Corollary 10 Let $X$ and $Y$ be two random variables with distribution functions $F$ and $G$, respectively, such that $X \leq_{e w} Y$. If $G(X)=G(Y)$, then $X \equiv_{s t} Y+c$, for some real constant $c$.

Proof. Note that $G(X)$ is given by (4) with $A(t)=t^{2}$ and $B(t)=$ $t$ (hence $\alpha(t)=2 t$ ). Therefore, the result is a immediate consequence of Theorem 9 .

If $X$ and $Y$ are non-negative random variables, Corollary 10 can be extended as follows:

Corollary 11 Let $X$ and $Y$ be two non-negative random variables with distribution functions $F$ and $G$, respectively, such that $X \leq_{e w} Y$. If

$$
E\left[X_{n: n}-X_{n-1: n}\right]=E\left[Y_{n: n}-Y_{n-1: n}\right]
$$

for some $n$, then $X \equiv_{s t} Y+c$, for some real constant $c$.

Proof. Since $E\left[X_{n: n}-X_{n-1: n}\right]$ is given, up to a scale parameter, by (4) with $A(t)=t^{n}$ and $B(t)=t^{n-1}$ (hence $\alpha(t)=\frac{n t}{n-1}$ ). Therefore, the result follows from Theorem 9 .

An analogous result can be stated in terms of Wang's right-tail deviation, noted by $D(X)$. 
Corollary 12 Let $X$ and $Y$ be two random variables with distribution functions $F$ and $G$, respectively, such that $X \leq_{e w} Y$. If $D(X)=D(Y)$, then $X \equiv_{s t} Y+c$, for some real constant $c$.

Proof. Wang's right-tail deviation belongs to $C$ with $A(t)=1-\sqrt{1-t}$ and $B(t)=t$ (therefore $\alpha(t)=1 / 2 \sqrt{1-t}$ ). The result now follows applying Theorem 9 .

\section{Conclusions and final remarks}

In this paper, we have characterized two nested classes of risk measures, $C_{1}$ and $C_{2}$, in terms of dispersive order and excess wealth order, respectively. The main result, Theorem 8, can be useful in empirical studies: the class $C_{1}$ contains a multitude of measures and it is impossible to verify directly the inequality

$$
\rho_{A, B}(X) \leq \rho_{A, B}(Y) \text {, for all } \rho_{A, B} \in C_{1} .
$$

Because $X \leq_{\text {disp }} Y$ is sometimes easy to verify (Shaked and Shanthikumar, 2006, point out several simple conditions that imply dispersive order), part (i) of Theorem 8 provides us with an easy way to check (32). In addition, part (ii) of Theorem 8 shows that if dispersive order is not available, unanimous preferences are still possible by restricting our attention to the class $C_{2}$, which is more sensitive to the variability within the right-tail of the distribution, and then the criterion is the excess wealth order.

A subclass of $C_{2}$ of particular interest is

$$
C_{3}=\left\{\rho_{A, B} \in C_{2}: B(t)=t\right\}
$$

which turn out to be the class of risk measures (3) suggested by Wang (1998). Ramos and Sordo (2003) characterized this class in terms of the well-known dilation order (recall that $Y$ is more dispersed than $X$ in the dilation sense, denoted by $X \leq_{d i l} Y$, if $E\left[\Phi\left(X-\mu_{X}\right)\right] \leq E\left[\Phi\left(Y-\mu_{Y}\right)\right]$ for all convex functions $\Phi$, provided that these expectations exist) as follows:

$$
X \leq_{\text {dil }} Y \text { if, and only if, } \rho_{A, B}(X) \leq \rho_{A, B}(Y) \text { for all } \rho_{A, B} \in C_{3} .
$$

The two-sided deviation suggested by Wang (1998) and the expected shortfall at level $p$ are measures of $C_{1}$ not belonging to $C_{3}$; in addition, (5), for $n \geq 2$, is a family of measures of $C_{2}$ that not belongs to $C_{3}$. These 
examples show that not every measure of interest of $C_{1}$ and $C_{2}$ belongs to $C_{3}$ and justify the convenience of employing dispersive order and excess wealth order rather than the weaker dilation order to compare risks.

We have also derived sufficient conditions, in terms of some special risk measures of the class $C_{2}$, for stochastic equality of two excess-wealth ordered random variables. These conditions have interesting statistical applications. For example, results of Section 3 can be applied to develop suitable tests of equality of two excess wealth ordered random variables. Specifically, if we consider the problem of testing the null hypothesis

$$
H_{0}: X \equiv_{s t} Y+c, \text { for some constant } c>0
$$

against the alternative

$$
H_{1}: X<_{e w} Y
$$

given random samples of $X$ and $Y$, we may use as a measure of departure from $H_{0}$ in favor of $H_{1}: \Delta=\left\{E\left|Y_{1}-Y_{2}\right|-E\left|X_{1}-X_{2}\right|\right\}$, where $X_{1}, X_{2}$ (resp. $Y_{1}, Y_{2}$ ) are independent copies of $X$ (resp. $Y$ ). Corollary 10 suggests reject the null hypothesis if $\Delta(n, m)>i_{0}$, where $\Delta(n, m)$ is an estimator of $\Delta$ based on two random samples $\left(x_{1}, \ldots, x_{n}\right)$ and $\left(y_{1}, \ldots, y_{m}\right)$ from $X$ and $Y$, respectively, and $i_{0}$ depends on the null distribution of $\Delta(n, m)$.

\section{Aknowledgement}

I am grateful to the referee for helpful comments.

\section{References}

Bhattacharjee, M.C., 1991. Some generalized variability orderings among life distributions with reliability applications. Journal of Applied Probability 28, 374-383.

Bhattacharjee, M.C., Bhattacharya, R.N., 2000. Stochastic equivalence of convex ordered distributions and applications. Probability in the Engineering and Informational Sciences 14, 33-48.

Bhattacharjee, M.C., Sethuraman, J., 1990. Families of life distributions characterized by two moments. Journal of Applied Probability 27, 720-725. 
Belzunce, F., 1999. On a characterization of excess wealth order by the increasing convex order. Statistics and Probability Letters 45, 103-110.

Bickel, P.J., Lehmann, E.L., 1979. Descriptive statistics for nonparametric models IV. Spread. In: Jureckova (Ed.), Contributions to Statistics. Reidel, Dordrecht.

Cai, J., Wu, Y., 1997. Characterization of life distributions under some generalized stochastic orderings. Journal of Applied Probability 34, $711-719$

Chateauneuf, A., Cohen, M., Meilijson., I., 2004. Four notions of meanpreserving increase in risk, risk attitudes and applications to the rankdependent expected utility model. Journal of Mathematical Economics $40,547-571$.

Chateauneuf, A., Cohen, M., Meilijson., I., 2005. More pessimism than greediness: a characterization of monotone risk aversion in the rankdependent expected utility model. Economic Theory 25, 649-667.

Denuit, M., Lefèvre, C., Shaked, M., 2000. On the theory of high convexity stochastic orders. Statistics and Probability Letters 47, 287-293.

Dhaene, J., Vanduffel, S., Goovaerts, M.J., Kaas, R., Tang, Q., Vyncke, D. 2006. Risk measures and comonotonicity: a review. Stochastic Models $22,573-606$.

Dhaene, J., Laeven, R., Vanduffel, S., Darkiewicz, G., Goovaerts, M.J., 2007. Can a coherent risk measure be too subadditive? Journal of Risk and Insurance. To appear.

Fernández-Ponce, J.M., Kochar, S.C., Muñoz-Perez, J., 1998. Partial orderings of distributions based on right-spread functions. Journal of Applied Probability 35, 221-228.

Goovaerts, M.J., Kaas, R., van Heerwaarden, A.E., Bauwelinckx, T., 1990. Effective Actuarial Methods. North-Holland, Amsterdam.

Jones, B.L., Zitikis, R., 2003. Empirical estimation of risk measures and related quantities. North American Actuarial Journal 7 , 44-54. 
Jones, B.L., Puri, M.L., Zitikis, R., 2006. Testing hypotheses about the equality of several risk measure values with applications in insurance. Insurance: Mathematics and Economics 38, 253-270.

Jones, B.L., Zitikis, R., 2007. Risk measures, distortion parameters, and their empirical estimation. Insurance: Mathematics and Economics 41, $279-297$.

Jun, C., 1994. Characterizations of life distributions by moments of extremes and sample mean. Journal of Applied Probability 31, 148-155.

Kaas, R., van Heerwaarden, A.E., Goovaerts, M.J., 1994. Ordering of Actuarial Risks. Caire Education Series: Amsterdam

Kendal, M., Stuart, A., 1977. The advanced Theory of Statistics, Vol. 1. Distribution Theory, 4th Edition. Charles Griffin and Company, London.

Kochar, S.C., Carrière, K.C., 1997. Connections among various variability orderings. Statistics and Probability Letters 35, 327-333.

Kochar, S.C., Li, X., Xu, M., 2007. Excess wealth order and sample spacings. Statistical Methodology, doi:10.1016/j.stamet.2006.11.002

Landsberger, M., Meilijson, I., 1994. Comonotone allocations, Bickel-Lehmann dispersion and the Arrow-Pratt measure of risk aversion. Annals of Operations Research 52, 97-106.

Landsberger, M., Meilijson, I., 1994. The generating process and an extension of Jewitt's location independent risk concept, Management Science 40, 662-669.

Li, X., 2005. A note on the expected rent in auction theory. Operation Research Letters 33, 531-534.

Li, H., Zhu, H., 1994. Stochastic equivalence of ordered random variables with applications in reliability theory. Statistics and Probability Letters 20, 383-393.

Muñoz-Pérez, J. 1990. Dispersive ordering by the spread function. Statistics and Probability Letters 10, 407-410. 
Nygard F., Sandström, A., 1981. Measuring income inequality. Almqvist \& Wiksell International. Stockholm, Sweden.

Quiggin, J., 1982. A theory of anticipated utility. Journal of Economic Behavior and Organization 3, 323-343.

Ramos H.M., Sordo M.A., 2003. Dispersion measures and dispersive orderings. Statistics and Probability Letters 61, 123-131.

Rockafellar, R.R., Uryasev, S., Zabaranking, M., 2006. Generalized deviations in risk analysis. Finance and Stochastics 10, 51-74.

Scarsini, M., Shaked, M., 1990. Some conditions for stochastic equality. Naval Research Logistics Quarterly 37, 617-625.

Shaked, M., Shanthikumar, J.G., 1998. Two variability orders. Probability in the Engineering and Informational Sciences 12, 1-23.

Shaked M., Shanthikumar J.G., 2006. Stochastic orders. Series: Springer Series in Statistics, Springer.

Sordo, M.A., Ramos, M.A., 2007. Characterizations of stochastic orders by L-functionals. Statistical Papers 48, 249-263.

Wang, S., 1996. Premium calculation by transforming the layer premium density. ASTIN Bulletin 26, 71-92.

Wang, S., 1998. An actuarial index of the right-tail risk. North American Actuarial Journal 2, 88-101. 